\title{
Application of Long-Acting Communication Mechanism in Improving nursing staff satisfaction in the Emergency Department
}

\author{
Sang Ziyang $^{1^{*}} \quad$ Luo Yumei $^{2}$ Huang Jianmao ${ }^{3}$ Huang Xuanjie ${ }^{4 \#}$
}

\begin{abstract}
${ }^{1}$ Emergency Department, the Seventh Affiliated Hospital, Sun Yat-sen University, Shen Zhen,518107; Sang Ziyang, Email:270928814@qq.com,https://orcid.org/0000-0003-3519-7344

${ }^{2}$ Emergency Department, the Seventh Affiliated Hospital, Sun Yat-sen University, Shen Zhen,518107; Luo Yumei, Email:1530578056@qq.com

${ }^{3}$ Emergency Department, the Seventh Affiliated Hospital, Sun Yat-sen University, Shen Zhen,518107; Huang Jianmao, E-mail:274211678@qq.com

${ }^{4}$ Emergency Department, the Seventh Affiliated Hospital, Sun Yat-sen University, Shen Zhen,518107; Huang Xuan-jie, E-mail: 190829610@qq.com, https://orcid.org/0000-0002-8811-7937

* Sang Ziyang is the first authors. \# Huang Xuanjie is the corresponding author.
\end{abstract}

Article History Received 26 August 2020 Accepted 25 September 2020 Published 31 December 2020

Cite this Article Sang Ziyang, Luo Yumei, Huang Jianmao, Huang Xuanjie. Application of Long-Acting Communication Mechanism in Improving nursing staff satisfaction in the Emergency Department [J].Medical Research, 2020.2(4):16-22, http://dx.doi.org/10.6913/MRHK.202012_2(4).0003

Copyright (C)2020 Creative Publishing Co., Limited.All rights reserved.Email:mrhk26640333@gmail.com.

\section{ABSTRACT}

Objective To explore the application of long-acting communication mechanism in improving nursing staff satisfaction in the emergency department.

Methods Since January 2020, the nursing management plan of the emergency department has been adjusted, a multi-channel and multi-departmental communication platform has been established, and a long-term communication mechanism involving all staff in the emergency department has been required to improve the quality of nursing work and improve the satisfaction of emergency department employees and patients.

Results After adjustment, the enthusiasm of nursing staff in emergency department were significantly improved, and the satisfaction of nursing staff was significantly improved $(\mathrm{P}<0.05)$.

Conclusion The application of long-term communication mechanism in emergency department nursing management can significantly improve the quality of nursing management, so as to effectively improve the satisfaction of nurses with the work.

Keywords emergency department; long-acting communication; satisfaction; nurse

The essence of long-term communication mechanism is the communication of ideas, views and emotions. By creating an open and transparent communication atmosphere, maintaining the smoothness of communication channels and improving communication satisfaction ${ }^{[1]}$, The nursing staff bears many tasks in the management of emergency department. If there is no long-term communication mechanism, the nursing 
Sang Ziyang, et al. Application of Long-Acting Communication Mechanism in Improving nursing staff satisfaction Medical Research ISSN 2664-0333 eISSN 2664-0341 Volume 2 Issue 4 http://dx.doi.org/10.6913/MRHK.202012_2(4).0003

staff cannot timely and effectively reflect the problems and solve the difficulties, which will seriously affect the work satisfaction of the nursing staff.

The emergency department is a multidisciplinary and multidimensional department, which involves the contents of internal and external, gynecological, pediatric and health care and security. It requires the exchange and cooperation of multiple departments, multiple departments and social organizations. If the communication is not smooth, it will cause many inconveniences, thus affecting the work quality and work efficiency of emergency emergency nursing. The subjects and objects of clinical and administrative management are relatively independent groups or individuals, so in each link of the management process, there is a need to communicate between the subjects and objects, and run through the whole process of management practice. Communication has become the main way, method and means to achieve various management objectives, and emergency patients are in critical, serious, complex and inaccessible condition, so that the emergency department medical staff have heavy work tasks, high pressure, irregular life, long-term stress state, there is no good long-term communication channel will seriously reduce the work satisfaction of nurses ${ }^{[2]}$.

This study found that after the establishment of a long-term communication mechanism, the quality of nursing work was significantly improved, and the satisfaction of nursing staff was also significantly improved, as reported below.

\section{Materials and Methods}

\subsection{General information}

Forty nursing staff in the emergency department of the Seventh Affiliated Hospital, Sun Yat-sen University from January 2020 to December 2020, aged $22 \sim 48$ years, participated in the work for $1.5 \sim 33$ years, were studied. Education level: 1 graduate student, 39 undergraduate; professional title: 1 deputy chief nurse, 5 chief nurses, 20 nurses, 14 nurses. By adopting a long-term communication mechanism, the satisfaction of general practice nursing staff in family life, accommodation, transportation, work environment, scheduling, promotion, performance, friendship administrative logistics communication, and doctor-patient communication was compared before and after.

\subsection{Method}

1.2.1 The problems existing in the work of emergency department nursing staff are widely collected by various means such as letter box, opinion form, symposium and network, and questionnaire. Managers should effectively communicate with employees to understand their needs through effective communication, including salary and treatment, employee welfare, rest system, training and development, etc.; understand the needs of employees for each link, formulate human resources management policies and personnel management system based on employee needs, and mobilize employee enthusiasm ${ }^{[3]}$. Through the collected data, it was found that the main problems of nursing staff were as follows: family life, accommodation, transportation, work environment, scheduling, promotion, performance, doctor-patient communication, and interdepartmental communication (including clinical friendship and administrative logistics departments). Each indicator was investigated and scored (very dissatisfied $=1$, dissatisfied $=2$, fair $=3$, satisfied $=4$, very satisfied $=5$ using the Likert 5-level scoring method) to calculate a total score. The minimum score is 9 and the full score is 45 .

1.2.2 Classify and sort out the problems: After collecting the problems, through specially-assigned sorting out, the problems can be divided into three main aspects: clinical work, relay learning and scientific research, and family life. Through democratic discussion, select three experienced and helpful colleagues from nursing staff as corresponding assistance secretaries, carry out one-on-one communication and handling according to the actual situation, timely report the situation to head nurses and department directors according to the difficulty of the problem, and invite relevant departments of the hospital to cooperate in solving the problem when necessary, so as to ensure that nursing colleagues can timely reflect the problem and effectively solve 
Sang Ziyang, et al. Application of Long-Acting Communication Mechanism in Improving nursing staff satisfaction Medical Research ISSN 2664-0333 eISSN 2664-0341 Volume 2 Issue 4 http://dx.doi.org/10.6913/MRHK.202012_2(4).0003

the problem. The three secretaries collate various problem-solving processes, methods, and ideas quarterly and share them in democratic meetings and nursing groups, so that each nursing colleague can clearly know the ways and methods to solve the problem.

1.2.3 Construction of long-term communication mechanism and platform: 1) WeChat group: including nursing group, medical and nursing work group; 2) nursing exchange meeting; 3) medical and nursing backbone safety meeting; 4) general practice meeting; 5) democratic life meeting; 6) secretary group. Each group or management organization shall establish secretaries specially collecting and sorting materials, and integrate and classify the problems. Meetings are held in a timely manner and tracked on an ongoing basis according to time urgency and ease. Treatment principle: Promote the principle of immediate solution, on-site treatment that can be immediately solved, so as to avoid negotiation. For problems that cannot be solved immediately, it is required to solve them within one week. For individual difficult problems that cannot be completed within one week, the reasons shall be explained and the schedule and corresponding support system shall be listed.

1.2.4 Hold a general meeting of all medical staff, carry out communication skills training, and announce the purpose and role of establishing each WeChat group or organization, so as to achieve fairness, impartiality and rationality, so that it can truly play the corresponding role. Let each nursing staff have a suitable platform to be able to express their thoughts and reflect their situation in a timely and effective manner.

\subsection{Specific operation method}

1.3.1 Family, life, transportation issues

Nursing staff work pressure, daily affairs are complex, need to pay a lot of energy in the work, but to deal with the work, we still need to face a lot of problems that must be treated with care. Through the collection of questionnaires, field visits, and democratic centralized meetings, it was found that most of the nursing staff had troubles in the care, transportation inconvenience, and housing problems of children and women in the family. In these things, it takes not only a lot of time, but also a lot of energy. In order to improve the work efficiency of nursing staff, under the organization of the director of the department and the head nurse, several solutions have been discussed in a centralized manner: 1) Advocate the spirit of mutual assistance, organize colleagues to hold family friendship activities, be familiar with each other, enhance friendship, and do a good job in mutual assistance in family care, children's school delivery, etc. 2) In terms of transportation, organize volunteer convoys, release downwind vehicle service information at the time, use shared vehicles to solve the travel problems of colleagues; apply to the hospital for commuting shuttles; it is recommended that the hospital apply to the transportation department for increasing the bus route of the hospital to facilitate the commuting of employees. 3) The personnel of the department shall be responsible for the relevant work of public rental application, assist the new colleagues in the relevant matters of public rental application; give nurses rental subsidies and improve welfare benefits. On the other hand, provide nursing colleagues with a variety of feedback channels, so that nurses can fully express their subjective wishes and needs, and the department can solve them in a timely manner according to the actual situation and relevant regulations.

\subsubsection{Work environment}

Workplace violence is an important factor leading to empathy fatigue in nursing staff. Violence can be divided into two types: psychological and physical. Psychological violence caused by abuse and threat is more common but easy to be ignored by managers. In addition, horizontal violence between colleagues is more likely to trigger psychological stress disorders and aggravate occupational burnout in nursing staff than violence from patients and their families ${ }^{[4]}$. When caregivers are in an unsafe working environment and are not recognized by patients and families, the compassion for patients is reduced ${ }^{[5,6]}$. The quality of the work environment directly affects the work efficiency of nursing colleagues and enthusiasm for work. In order to improve the above environmental problems, the department gradually improves the satisfaction of medical 
Sang Ziyang, et al. Application of Long-Acting Communication Mechanism in Improving nursing staff satisfaction Medical Research ISSN 2664-0333 eISSN 2664-0341 Volume 2 Issue 4 http://dx.doi.org/10.6913/MRHK.202012_2(4).0003

staff through democratic discussion and decision, from strengthening security, installing one-touch alarm and anti-wolf spray in each area to the renovation of workplace environment, division of special treatment area for intoxicated trouble, update of work instruction identification, maintenance of environmental sanitation and invitation to guard regular to emergency training and guidance and organizing joint drills.

\subsubsection{Scheduling}

The emergency department has large areas, many personnel, and diverse needs, and there are great difficulties in scheduling. The previous use of manual scheduling methods is prone to some unreasonable arrangements or missed scheduling of a certain position, and nurses have more complaints. In today's high informatization, the application of scheduling small procedures makes the benign operation of nursing management on a standardized and scientific track increasingly important ${ }^{[7]}$. It conforms to the humanized concept of modern management, fully respects the dominant position of nurses, takes meeting the psychological needs of nurses as the first priority, not only mobilizes the accumulation polarity of nurses, greatly stimulates the work enthusiasm of nurse groups, improves work efficiency and nursing quality, but also creates a relaxed and harmonious work atmosphere, closes the distance between nursing managers and frontline nurses, and obtains a win-win situation ${ }^{[8]}$. Currently, scheduling is performed by using a new scheduling software. The new scheduling software can record the needs and expected shifts of nursing colleagues in the software, which better avoids the occurrence of missed and wrong scheduling shifts. In addition, nurses can also transfer shifts through software, which is convenient, quick and effective through software information transmission, facilitates efficient communication between managers and nurses, and reduces time costs.

\subsubsection{Promotion, Performance}

Promotion is related to the career and salary issues of each caregiver. For this year's issues requiring promotion, the captain organizes and convenes a symposium of colleagues for promotion to widely listen to and collect relevant issues and opinions. Organize the relevant scientific research, teaching, and clinical nursing secretaries for corresponding follow-up guidance, and organize an effect evaluation meeting once a month to ensure that each promoted nursing colleague can pass with excellent results by the end of the year.

Performance is a concern for all colleagues and a driving force for everyone to work. In terms of this year's performance evaluation, the department held a performance evaluation meeting of the general practice according to the actual situation of the hospital and the department, interpreted the relevant documents on the development of the hospital and the department, allowed all colleagues to participate in the formulation and modification of the performance according to the spirit of the document, and required everyone to actively speak and put forward corresponding suggestions. After the performance evaluation plan comes out, it will be timely published, requiring each colleague to carefully read and understand, timely put forward doubts, achieve impartiality, fairness and openness, and ensure that each colleague can accept and understand them. 1.3.5 Clinical Department and Administrative Logistics Department Communication

The emergency department is the window of spiritual civilization of the hospital, which involves multiple fields and needs to communicate and coordinate relevant matters with various clinical departments and administrative logistics departments. Therefore, good communication and cooperation can improve work efficiency and increase employee satisfaction. The department director and head nurse should regularly collect the problems occurred in clinical work and deal with them in a timely manner. If necessary, conduct joint office work of multiple departments and departments, and solve the problem as soon as possible.

\subsubsection{Doctor-patient communication}

The characteristics of high risk and acute condition of patients in emergency department require nurses to have the ability of communication and comprehension to quickly understand the patient's condition, so as to quickly give the corresponding rescue measures. The highest score among the three dimensions of empathy was point selection, and the scores of emotional care and empathy were low, indicating that clinical nurses can 
accept the psychological views of patients at present, but it is difficult to resonate with patients and give emotional care. The front-line clinical work is busy, nurses often focus on completing the nursing operation task, and do not have enough time to accurately identify and accept various emotions and emotions of patients. Yang $\mathrm{Xi}$ et al ${ }^{[9]}$. suggested that the lack of empathy training and the limitation of busy work are the reasons for the low level of empathy in clinical nurses. A survey of the work status of nurses nationwide by Xu Yimin et $\mathrm{a}^{[10]}$. showed that $74.73 \%$ of nurses believed that their work pressure was high or large, and they faced psychological pressure in the face of clinical nurses in tertiary hospitals with more complex and onerous work in the front line of clinical practice, which may lead to a reduction in their ability to empathize with patients. The head nurse of the department has optimized the allocation of human resources, paid attention to the psychological adjustment of clinical nurses, carried out empathy training by referring to successful experiences such as narrative nursing education and digital stories, improved the empathy level of clinical nurses, and promoted the smooth development of clinical nursing work. The emergency department establishes an emergency department doctor-patient communication group in early 2020, which collects corresponding information every day and continuously tracks the problem. Carry out democratic life meetings every month, listen to the voices of nurses, and solve the problems raised by nursing staff in a timely manner, so as to improve nursing staff satisfaction.

\subsubsection{Evaluate the operation of long-term communication mechanism}

In order to better achieve the expected reform objectives, public institutions need to continuously adjust and optimize in internal management ${ }^{[11]}$. Every month, we should effectively collect receipts and reflect the operation and actual effect of each platform from different levels. We should fully improve the enthusiasm of nursing staff to respond to problems, thus also supervise the effectiveness and timeliness of the mechanism in dealing with problems, continuously adjust and optimize the operation of the communication mechanism, listen to the opinions of personnel at different posts and levels, widely absorb everyone's suggestions, so that this long-term communication mechanism can operate steadily and effectively.

\section{Results}

2.1 Comparison of caregiver satisfaction before and after implementation (\%)

\begin{tabular}{ccc}
\hline Item & \multicolumn{2}{c}{ Item } \\
\cline { 2 - 3 } & $\begin{array}{c}\text { Before } \\
\text { implementation }\end{array}$ & $\begin{array}{c}\text { After } \\
\text { implementation }\end{array}$ \\
\hline Satisfaction\% & 80 & 95 \\
Cassette value & \multicolumn{2}{c}{4.114} \\
P value & \multicolumn{2}{c}{0.04} \\
\hline
\end{tabular}

Table 1

2.2 Comparison of various evaluation indicators before and after implementation $(\mathrm{x} 2 \pm \mathrm{s})$

\begin{tabular}{ccccc}
\hline Item & $\begin{array}{c}\text { Before } \\
\text { implementation }\end{array}$ & $\begin{array}{c}\text { After } \\
\text { implementation }\end{array}$ & T value & P value \\
\hline $\begin{array}{c}\text { Work } \\
\text { environment }\end{array}$ & $2.650 \pm 0.622$ & $4.075 \pm 0.572$ & -10.679 & 0.000 \\
Scheduling & $2.350 \pm 0.769$ & $3.975 \pm 0.767$ & -9.528 & 0.000 \\
Promotion & $2.400 \pm 0.708$ & $3.650 \pm 0.769$ & -7.486 & 0.000 \\
Performance & $2.600 \pm 0.671$ & $3.900 \pm 0.871$ & -6.500 & 0.000 \\
Assignment & & &
\end{tabular}

Table 2

\section{Discussion}


Nurses are at high risk of occupational burnout. Long-term high-intensity and high-stress work is prone to physical fatigue, emotional exhaustion and reduced sense of work accomplishment, and the risk of occupational burnout is high ${ }^{[2]}$, which will lead to a reduction in the satisfaction with their own work. After the nursing staff has the way and method to reflect various problems by setting up a variety of communication channels, the satisfaction of nursing colleagues with the work is increased from $80 \%$ before the implementation to $95 \%$ after the implementation (Table 1), and the satisfaction in terms of specific work environment, scheduling, promotion, and performance distribution is also significantly improved (see Table 2 for details). Therefore, by establishing a long-term communication mechanism and channel, the nursing staff can timely reflect the clinical, learning and life problems, and solve them in a timely manner, so that their whole-body investment in the work can effectively improve their satisfaction with their own work.

\section{REFERENCES}

[1] Zhao Xiaobo. Research on Office Work Innovation of College Based on Long-lasting Communication Mechanism [J]. China Management Informatization, 2017, 20 (06): 210-211.

[2] Zhang Wei, Chen Yonghui, Meng Lijuan. Investigation and Analysis of Job Stress, Coping Style and General Self-Efficacy of Nurses in Emergency Department [J]. Chinese Journal of Practical Nursing, 2016, 32 (14): 1041-1044.

[3] Liu Junhui. Effective Communication and Internal Human Resource Marketing [J]. China Management Informatization, 2020, 23 (20): 122-123.

[4] Fan Si, Yi Qifeng, Kang Liyang, Huang Hui. Research progress of stress disorder and its influencing factors after nurses encounter workplace violence [J]. Chinese Journal of Nursing, 2018, 53 (12): 1451-1454. [5] Wang Xue, Li Ling. Effect of workplace violence and job burnout on empathy fatigue of nurses in emergency department [J]. Journal of Nursing, 2019, 34 (06): 58-61.

[6]Chizimuzo T. C. Okoli PhD, MPH, MSN, RN, Sarret Seng BA, BSN, RN, Janet K. Otachi BSW, MA, et al. A cross-sectional examination of factors associated with compassion satisfaction and compassion fatigue across healthcare workers in an academic medical centre. 2020, 29(3):476-487.DOI:10.1111/inm.12682.

[7] Jiang Yan, Lao Lixia, Peng Dongwei, Tan Huiyi, Li Meihua. Using Computer Network to Manage Nurse Scheduling [J]. International Herald of Medicine and Health, 2003 (16): 130-131.

[8] Ma Sensitive. Application Practice of Mobile Nursing Scheduling System Based on Smartphone APP [J]. Nursing Research, 2018, 32 (10): 1653-1655.

[9] Yang Xi, Shi Ruifen. Research Status and Prospect of Nurses' Empathy Ability [J]. Journal of Nursing, 2012, 27 (16): 86-89.

[10] Xu Weimen, Wu Ying, Zhang Yan, Ma Ruiying, Li Xiuhua. Investigation and analysis of the working status of nurses in hospitals in China [J]. Chinese Journal of Nursing, 2016, 51 (08): 947-950.

[11] Wu Yanqing. On the Present Situation and Improvement Thinking of the Performance Evaluation Mechanism of Public Institutions [J]. China Collective Economy, 2019 (21): 50-51.

[12] Liu Chenliu, Guo Yanfeng, Li Huanmei, Liu Qingyan, Zhu Jing, Deng Qiuying. Psychological flexibility, job burnout and job satisfaction of 200 emergency nurses in Guangzhou [J]. Industrial Hygiene and Occupational Diseases, 2019, 45 (04): 244-247. 